\title{
GASTRO RETENTIVE DRUG DELIVERY SYSTEM: A SIGNIFICANT TOOL TO INCREASE THE GASTRIC RESIDENCE TIME OF DRUGS
}

\author{
KANUPRIYA C., NIMRATA SETH, N. S. GILL \\ Department of Pharmaceutics, Rayat Institute of Pharmacy, Railmajra (Ropar), Punjab, India \\ Email: Kanupriya176@gmail.com
}

Received: 18 Sep 2020, Revised and Accepted: 17 Nov 2020

\begin{abstract}
Writing the review on gastro retentive drug delivery systems (GRDDS) was to start up the current literature with a special consequence on several gastro retentive approaches that have become main mode in the field of site-specific orally conduct sustained/controlled release drug delivery. Multi-furious ways have been made in research and development to rate-controlled oral drug delivery systems to solve physiological difficulties, like short gastric residence times (GRT) and unpredictable gastric emptying times (GET). GRDDS is a tool to prolong the GRT, thereby targeting sitespecific drug release in the upper gastrointestinal tract (GIT) for local or systemic effect. Oral dosage forms as low bioavailability issues because of their swift gastric transition from the stomach, particularly in the case of drugs that are less soluble at an alkaline $\mathrm{pH}$ of the intestine. The drugs that produce their local action in the stomach get quickly emptied and don't get enough residence time in the stomach. Many efforts have been made to extend the retention time of a drug delivery system to reduce the frequency of dose administration. GRDDS not only prolong dosing intervals but also increase patient compliance beyond the level of existing controlled release dosage forms. This article gives an overview of the advantages, disadvantages, and characterization of gastro retentive drug delivery systems. This also includes commercially available gastro retentive products and patents.
\end{abstract}

Keywords: Gastro retentive drug delivery, Upper gastrointestinal tract, Gastrointestinal, Local or systemic effect, Alkaline $\mathrm{pH}$ of the intestine, Retention time

(C) 2021 The Authors. Published by Innovare Academic Sciences Pvt Ltd. This is an open access article under the CC BY license (https://creativecommons.org/licenses/by/4.0/) DOI: https://dx.doi.org/10.22159/ijcpr.2021v13i1.40818. Journal homepage: https://innovareacademics.in/journals/index.php/ijcpr

\section{INTRODUCTION}

Oral drug delivery system (ODD) and convenient route of drug administration due to high patient compliance, cost-effectiveness, least sterility constraints, flexibility in the design of dosage form and ease of production. The oral delivery system is as some disadvantages as poor bioavailability of the drug, which is determined by three vital factors, namely, dissolution, permeability, and solubility. Oral controlled release drug delivery. It is mostly used in the pharmaceutical field and improved therapeutic such as ease of dosing administration, patient compliance, and flexibility in formulation. Due to excessive curative benefits of the oral controlled release, dosage forms are being preferred as the interesting topic of research over the past 3 decades. The much obvious interest in this scenario is due to its two-fold advantage. Primarily, the oral controlled release dosage forms have the potential to upkeep an effective concentration in the system for a longer duration. Oral controlled release drug sustains the virtual concentration in the body but due to its short gastric retention time and short gastric emptying time. It improves bioavailability, prolongs the duration of drug release, reduces drug waste, and ameliorate the drug solubility that is less soluble in a high $\mathrm{pH}$ environment. Also, prolonged gastric retention time (GRT) in the stomach could be benignant for local action in the upper part of the small intestine e. g. treatment of peptic ulcer, etc. Early To compensate for this effect, a very large dose is often steward so that absorption of therapeutically required quantity of drug can occur. This technique may prove costly with expensive drugs, and the absorbed drug may also have unwanted side effects within the gastrointestinal tract. Also, a poorly delivery system is developed, which increase the extent of the short half-life drugs. It can protect retaining ability in the stomach. This improves the gastric residence of drugs in the stomach. An atypical drug delivery system of gastro retentive dosage form has evolved. This problem may overcome by a modified release drug delivery system with prolonged residence time in the stomach [1]. Gastro retentive drug delivery system (GRDDS) is thus beneficial for such drugs by improving their bioavailability, therapeutic efficacy and by possible reduction of the dose. Gastro-resistant tablet dosage form is intentional to release a drug after some time that is a delay or after the tablet has passed through one part of the GI tract into another. Extended-release dosage forms are modified-release dosage forms showing a release of the active substance(s) that is slow. Enteric-coated dosage forms are designed to combat the acidic environment of the stomach and to fragment in the higher $\mathrm{pH}$ environment of the intestinal fluid. Proton pump inhibitors, $\mathrm{H}_{2}$ blockers, some NSAIDs, insulin delivery, etc are suitable candidates for developing delayed release dosage forms [2].

\section{Need for grdds}

Some drugs are absorbed and release at specific sites or a release such that the maximum amount of drug reaches to the specific site. The pharmaceutical field is focusing on such drugs that require sitespecificity $[2,3]$.

\section{Advantages of grdds}

An increase in bioavailability and curative efficiency of drugs and economic usage of dosage. Decrease factor of risk in resistance in antibiotics owing to stabilized therapeutic levels over prolonged periods removing fluctuations. Enhance release in case of short halflife drugs, causes flip flop pharmacokinetics and also ensures patient compliance with reduced dosage frequency. They are advantageous against drawbacks of the gastric retention time (GRT) as well as the gastric emptying time (GET). The system remains resilient on gastric fluid because of lower bulk density than gastric fluids. These are efficient in repairing the stomach and small intestine related problems. It applies to the fact that gastro retentive drug delivery sustains drug release and hence, benefits in local therapy in these organs. This method provides a systematic and controlled drug delivery system which decreases the chances of drug overexposure at the diseased site.

\section{Disadvantages of grdds}

Unsuitable for such drugs as: Problematic with solubility in gastric fluid. Causing G. I soreness. Inefficient in acidic environment. The ability of the drug to remain in the stomach depends upon the subject being positioned upright. Hydro gel-based swelling system takes a long time to swell. Upon multiple administrations, size increasing drug delivery systems pose a threat to life owing to the possible hazard of permanent retention in the stomach. Super porous systems having drawbacks like problematical storage of much easily hydrolyzable, biodegradable polymers [4]. 


\section{Physiology of stomach}

Structure: The stomach has four main regions. The main function of the Fundus and body is storage, whereas that of the cardiac is mixing or grinding. Fundus increased volume during eating by relaxation of the Fundus muscle fibers. It also exerts a steady pressure on the gastric contents pressing them towards the distal stomach. To pass through the pyloric valve in the small intestine, particles should be of the size of 1 to $2 \mathrm{~mm}$ called chime with the $\mathrm{pH}$ range is shown in table 2 [5-7].

Table 1: $\mathrm{pH}$ range

\begin{tabular}{ll}
\hline GIT & pH \\
\hline Stomach & $1-3$ \\
Small intestine & $5-7.5$ \\
Large intestine & $7.9-8$ \\
Rectum & $7.5-8$ \\
\hline
\end{tabular}

\section{Functions of the stomach}

The stomach carries three major functions. It stores food and convey food to the small intestine at a rate that the small intestine can handle. It's Acts as a reservoir for holding food before its release into the small intestine. It secretes gastric into the blood. It secretes gastric juice, which contains hydrochloric acid, pepsin, intrinsic factor, and gastric lipase mixes food and gastric juice to form chyme [8].

\section{Factors controlling gastric retention of dosage forms}

\section{Density}

GRT is a function of dosage form buoyancy that is dependent on the density and the dosage form also affects the gastric emptying rate. The dosage forms having a density lower than the gastric contents can float to the surface, while the high-density systems sink to the bottom of the stomach and the density of $<1.0 \mathrm{gm} / \mathrm{cm}^{3}$ is required to exhibit floating property of the dosage form [9-11].

\section{Size and Shape of dosage form}

Shape and size of the dosage forms are important in designing single indigestible unit solid dosage forms. In most cases, the larger the dosage form the greater will be the gastric due to the larger size of the dosage form would not allow this to quickly pass through the pyloric antrum into the intestine. Some devices such as Ring-shaped and tetrahedron shaped devices have a better gastric residence time as compared with other shapes. [12-14].

\section{Food intake and its nature}

The viscosity of food intake, and volume of food, caloric value and frequency of serving and have a deep effect on the gastric retention of dosage forms. The presence or absence of food in the GIT influences the gastric retention time (GRT) of the dosage form and thus, the drug absorption increases by allowing it to stay at the absorption site for a longer time. Again, an increase in acidity and caloric value shows down gastric emptying time (GET), which can improve the gastric retention of dosage forms.

\section{Effect of gender, posture, and age}

The gastric emptying rate is less in females as compared to males. The effect of posture does not have any significant difference in the mean gastric retention time (GRT) for individuals and supine state conditions. In the case of elderly persons, gastric emptying is slowed down [15-17].

\section{Regulation of gastric secretion and motility}

The two neural and hormonal mechanisms control the secretion of gastric juice along with the contraction of smooth muscles in the stomach wall. Events in gastric secretion occur in three overlapping phases; Cephalic phase, Gastric phase, and Intestinal phase.

\section{Cephalic phase}

The cephalic phase refers to the impact of the brain on secretion. Even before food enters the stomach, thought of food initiate this phase; the secretion is brought about through brace, of the nerve. This leads to the presence of acid and pepsin in the stomach even before food enters the stomach [18-20].

\section{Gastric phase}

The secretion is escorted by the presence of food in the stomach. It is managed by the hormone gastrin which is produced in the mucosa of the pyloric region of the stomach. Gastrin is released in response to stretching of the antrum caused by the presence of food in this region or response to specific substances in the food; particularly proteins, alcohol, and coffee are also stimulants of gastrin release.

\section{Intestinal phase}

The intestinal phase of acid secretion refers to the influence of the small intestine. If the material present in the duodenum of the small intestine is too acidic, a hormone is released by the intestinal mucosa. This hormone is carried out by the blood to the body of the stomach, where it prevents further acid secretion. This acts as a protective device for the small intestine, which is not as well protected against acid like the stomach. The total volume of gastric secretion in response to all the stimuli mentioned above is approximately 2 to $3 \mathrm{~L}$ per day.

\section{Gastric emptying}

The process of gastric emptying occurs during both fasting and fed state. In the fasted state, it is classified by an inter-digestive cycle both through the stomach and small intestine, every $2-3 \mathrm{~h}$. This is called the inter-digestive myoelectric cycle of migrating myoelectric complex (MMC). It is composed of four phases and table 2 [21-23].

Table 2: Phases of migrating myoelectric cycle or gastrointestinal dynamic

\begin{tabular}{lll}
\hline S. No. & Phase & Duration \\
\hline 1 & Phase 1 (basal phase) & $\begin{array}{l}\text { 30-60 min infrequent contraction } \\
\text { Phase last for 40-60 min with intermitted action potential and contraction, as the phase progress the } \\
\text { intensity and frequency also increase. }\end{array}$ \\
& Phase 2 (pre burst phase) & $\begin{array}{l}\text { Phase last for 4-6 min it includes intense contraction for short period it is also called housekeeper wave. } \\
\text { Phase last for 0-5 min and occur between phase 3andphase 4of the consecutive cycle }\end{array}$ \\
\hline
\end{tabular}

\section{Different approaches of grdds}

The concept of the gastro retentive drug delivery system was described in the literature as early as 1962 . It has a bulk density less than gastric fluids and so remains resilient in the stomach without arousing affect the gastric emptying rate for a prolonged period. The buoyancy on the gastric contents the drug is release slowly at the crave rate from the system and increased GRT and better control of fluctuations in plasma drug concentration. The device must have an acceptable structure to form a cohesive gel barricade, it must 
maintain an overall specific gravity lower than that of gastric contents (1.004-1.010) and should dissolve slowly enough to setup as a drug reservoir. Types of floating drug delivery systems Based on the mechanism of buoyancy and two distinctly different technologies have been utilized in the development of GRDDS.

\section{- Floating system}

- Non-floating system

- Floating system are classified as:

\section{Non-effervescent grdds}

\section{Effervescent grdds}

\section{Non-effervescent grdds}

The Non-effervescent GRDDS is based on the mechanism of swelling of polymer or bio adhesion to the mucosal layer in the GI tract such as polycarbonate, polyacrylate, polymethacrylate, polystyrene, Chitosan, and Carbopol. They are further classified as:

\section{Hydrodynamically balanced systems (HBS)}

These systems are designed to extend the stay of the dosage forms in the gastric-intestinal tract and aid in enhancing the absorption. Drugs having a good dispersible in acidic environment and also having specific site of absorption in the upper part of small intestine is achieved by these HBS systems. Occur in stomach for a prolonged period of time; the dosage form must have bulk density of less than ' 1 ' and has to maintain its structural integrity and release drug constantly from the dosage form.

\section{Single-layer floating tablets}

They are plan by the intimate mixing of drugs with low-density enteric materials such as CAP, HPMC.

\section{Bi-layer floating matrix tablets}

A bi-layer tablet means two-layer one prompt release layer which releases initial dose from the system while another sustained release layer absorbs gastric fluid, forming a dense colloidal gel obstruction on its surface, and maintain a bulk density of less than unity and thereby it remains buoyant in the stomach.

\section{Alginate beads multi-unit floating dosage forms}

Were formulated from freeze-dried calcium alginate. The beads which are spherical as minimal $2.5 \mathrm{~mm}$ diameter can be prepared by dropping sodium alginate solution into an aqueous solution of calcium chloride, causing precipitation of calcium alginate leading to the development of the permeable system, which can maintain a floating force for over $12 \mathrm{~h}$. When correlated with solid beads, which gave a short residence time of $1 \mathrm{~h}$, and these floating beads gave a prolonged residence time of more than $5.5 \mathrm{~h}$.

\section{Hollow microspheres}

Hollow microspheres (micro balloons), weighted with the drug in their outer polymer shells, are prepared by a novel emulsion-solvent diffusion method. The ethanol: dichloromethane solution of the drug and an enteric acrylic polymer is agitated with an aqueous solution of PVA that is thermally controlled at $40^{\circ} \mathrm{C}$. The gas-phase formed in diffuse polymer droplet by evaporation of dichloromethane forms an internal cavity in the microsphere of polymer with the drug. The micro balloons frequently float over the surface of acidic dissolution media containing surfactant for more than $12 \mathrm{~h}$ in vitro.

\section{Raft forming systems}

Have received much awareness for the delivery of antacids and drug delivery for gastrointestinal infections and disorders. Rafts floatation have been used in the treatment of Gastric esophageal reflux disease (GERDS). The mechanism used in the raft formation includes the formation of viscous cohesive gel in contact with gastric fluids, wherein each part of the liquid swells forming a continuous layer called a raft. Gastric fluids, because of low bulk density fabricated by the formation of $\mathrm{CO}_{2}$ habitually the system contains a gel-forming agent and alkaline bicarbonates or carbonates in levy of for the formation of $\mathrm{CO}_{2}$ to make the system less murky and float on the gastric fluids. An antacid raft setup floating system contains a gel forming agent (e. g. sodium alginate), sodium bicarbonate and acid counter, which forms a foaming sodium alginate gel (raft), which when comes in contact with gastric fluids, the raft floats on the gastric fluids and prevents the reflux of the gastric contents into the esophagus by acting as a barricade between the stomach and esophagus.

\section{Effervescent system}

Effervescent systems contain the use of gas generating agents, carbonates (ex. Sodium bicarbonate) and other organic acids (e. g. citric acid and tartaric acid) present in the system to produce carbon dioxide $\left(\mathrm{CO}_{2}\right)$ gas, thus decrease the density of the system and making it float on the gastric fluid. A different is the incorporation of a matrix containing a portion of liquid, which produces gas that evaporates at body temperature. These effervescent systems are further classified into two types.

\section{Gas generating systems}

\section{Volatile liquid/Vacuum containing systems}

\section{Gas generating systems (Tablets floating bilayer)}

Tablets with controlled release for furosemide were advanced by $\mathrm{Oz}$ emir et al., 2000. The low solubility of the drug could be increased by using the kneading method, preparing a solid dispersion with $\beta$ cyclodextrin mixed in a 1:1 ratio. One layer contained the polymers HPMC K4M, HPMC K100M, and CMC (for the extent of the drug delivery) and the drug. The second layer enclosed the effervescent mixture of sodium bicarbonate and citric acid. The in vitro floating studied enclosed that the lesser the compression force the shorter is the time of onset of floating, i.e., when the tablets were compressed at $15 \mathrm{MPa}$, these could begin to float at $20 \mathrm{~min}$ whereas at a force of $32 \mathrm{MPa}$ the time was increased to $45 \mathrm{~min}$. cathode rays. Studies on 6 healthy male volunteers show that floating tablets were retained in the stomach for $6 \mathrm{~h}$ and further blood analysis studies showed that the bioavailability of these tablets was 1.8 times that of the regular tablets. On measuring the volume of urine, the peak diuretic effect seen in the regular tablets was decreased and prolonged in the case of the floating dosage form.

\section{Floating capsules}

Are prepared by stuffing with a mixture of sodium alginate and sodium bicarbonate. The systems were shown to float during in vitro tests as a result of the generation of $\mathrm{CO}_{2}$ that was captured in the hydrating gel network on exposure to an acidic environment. Interactions occur between the complementary structures. However, these energies last only until the turnover process of mucin and, for a bioadhesive system to be successful.

\section{Multiple unit types floating pills}

The system contains sustained-release pills as 'seeds' surrounded by double layers. The inner layer is made up of effervescent agents while the outer layer is of the swellable membrane layer. When the system is an evince in dissolution medium at body temp, it drops at once and then forms expand pills like balloons, which float as they have lower density. This lower density is due to the generation and bait of $\mathrm{CO}_{2}$ within the system.

\section{A floating system with ion-exchange resins}

A floating system using ion exchange resin that was packed with bicarbonate by mixing the beads with $1 \mathrm{M}$ sodium bicarbonate solution. The packed pellets were then surrounded by a semipermeable membrane to avoid sudden loss of $\mathrm{CO}_{2}$. In gastric contents contain chloride and bicarbonate ions together form $\mathrm{CO}_{2}$ engendering, thereby carrying beads toward the top of gastric contents and mass-produce a floating layer of resin beads. The in vivo nature of the coated and uncoated pellets was observed using a single channel analyzing study in 12 healthy human volunteers by gamma radio scintigraphy. Studies showed that the gastric residence time was increased considerably (24 h) compared with uncoated beads ( 1 to $3 \mathrm{~h}$ ).

\section{Volatile liquid/Vacuum containing systems}

Intra-gastric floating gastrointestinal drug delivery system: In this system, it is for-gathered to float in the stomach because of 
floatation chamber, which may be a vacuum or filled with air, while drug reservoir is encapsulated inside a micro-porous.

\section{Inflatable gastrointestinal delivery systems}

Liquid ether is present in the inflatable chamber that gasify at body temperature to cause the chamber to inflate in the stomach. The inflatable chamber with a drug reservoir, which can be a drug impregnated polymeric matrix, encapsulated in a gelatin capsule. After oral conduct, the capsule dissolves to release the drug reservoir together with the inflatable chamber. The inflatable chamber no doubts, inflates and retains the drug reservoir compartment in the stomach. The drug is continuously released from the reservoir into the gastric fluid.

\section{Intragastric osmotically controlled drug delivery system}

In this device, an osmotic pressure-controlled drug delivery device and inflatable floating support in a biodegradable capsule. In the stomach, the capsule quickly releases the intragastric osmotically controlled drug delivery device. The expandable support inside forms a deformable hollow polymeric bag that contains a liquid that gasifies at body temperature to inflate the bag. The intragastric osmotic pressurecontrolled drug delivery device consists of two components; a drug reservoir compartment and an osmotically active compartment. The drug reservoir is enclosed by a pressure responsive collapsible bag, which is impermeable to vapors and liquid and has a drug delivery orifice. The osmotically effective compartment contains an osmotically active salt and is enclosed within a semipermeable housing. In the stomach, Gastric fluid is engaged through the semipermeable membrane into the osmotically active compartment to dissolve the osmotically active salt. The osmotic pressure builds acts on the collapsible bag and in turn, forces the drug reservoir compartment to reduce its volume and activate drug release through the delivery orifice. The floating may also contain a bio-erodible plug that is destroyed after a predetermined time to deflate the support and then emptied from the stomach.

\section{Non-floating system}

\section{Bioadhesive drug delivery system}

The term bio adhesion is defined as adhesion to biological surface i.e. mucus and/or mucosal surface. Incite when the polymeric system interacts with the mucus layer only, it is referred to as muco adhesion. An oral bioadhesive system, it is important to have a thorough understanding of mucosa, bioadhesive polymers and mucin-polymer interactions in the physiological environment. Intestinal mucosa is unfazed of high molecular weight glycoproteins hydrated and covering the mucosa with a continuous adherent blanket. Mucin glycoproteins are rich with fucose and sialic acid groups at the fatal ends, which provide a net negative charge in the acidic environment. The density of the mucin gel layer varies in different regions of the GIT, with thickness ranging between 50$500 \mu \mathrm{m}$ in the stomach to $15-150 \mu \mathrm{m}$ in the colon. The coherence of the mucin gel is dependent upon the glycoprotein concentration. The mucus layer is generated biologically to play several functions of protecting the underlying tissues from various diffusing/corrosive elements such as enzymes, acid, and other toxic molecules. Also, being a viscos-elastic gel, it helps in the passage of food over the epithelium, thereby curtail potential erosive damages

\section{Swellable/Expandable system}

In this system which when enters to the stomach, swells (due to the presence of swellable polymers) to the extent that cannot pass through the pyloric sphincter leading to retained in the stomach for a long time referred to as "plug type systems" because they tend to remain lodged at the pyloric sphincter. Thus, three arrangements are required: Oral intake is swallowed in a small configuration. Expanded to a size that prevents their passage through the pylorus. Finally, after the drug release at a predetermined time, it becomes ready for an evacuation since the device is no longer can attain or retain the expanded configuration.

\section{Magnetic system}

These systems arrive as small gastro retentive capsules containing a magnetic material, whose eradication from the stomach is averted by the interaction with a sufficiently strong magnet enforced to the body surface in the suburb of the stomach. Despite many reports about successful tests, the real applicability of such systems is doubtful because the desired results can be achieved only provided that the magnet position is selected with very high precision. Probably, the development of a new technique applied to magnetic field sources will improve this concept. Self-unfolding systems the self-unfolding systems are capable of mechanically elevated in size relative to the initial dimensions. This increase prevents the system from passing via the pylorus and provides for its extended stay in the stomach. A drug can be either contained in a polymeric composition of the gastro retentive system or incorporator as a separate component. Several methods were found that provide for the self-unfolding effect: Hydrogels use as swelling in contact with the gastric juice. It is comprising an osmotic medium in a semipermeable membrane. Systems established on low-boiling liquids converting into a gas at the body temperature. Contents have a density near to water $\left(1.004 \mathrm{~g} / \mathrm{cm}^{3}\right)$. When the patient is upright position small high-density pellets sink to the bottom of the stomach where they become entrapped in the folds of the antrum and withstand the peristaltic waves of the stomach wall. A density close to $2.5 \mathrm{~g} / \mathrm{cm}^{3}$ seems important for the significant annex of gastric residence time and barium sulfate, zinc oxide, iron powder, titanium dioxide is used as excipients [24-26].

\section{High density (sinking) system}

Formulation of dosage forms with the density that must exceed the density of normal stomach content $\left(\sim 1.004 \mathrm{gm} / \mathrm{cm}^{3}\right)$. These systems are prepared by coating drug on a heavy elemental or mixed with inert materials such as iron powder, barium sulphate, zinc oxide and titanium oxide etc. The density rises by up to 1.5-2.4 $\mathrm{gm} / \mathrm{cm}^{3}$. A density close to $2.5 \mathrm{gm} / \mathrm{cm}^{3}$ seems paramount for significant prolongation of gastric residence time. But, the effectiveness of this system in human beings was not observed and no system has been marketed [28-30].

\section{Evaluation parameters of gastroretentive dosage forms}

\section{In vitro evaluation: general tests}

These tests include appearance, hardness, friability, drug content, weight Variation, uniformity of content.

\section{Floating system: Buoyancy lag time}

Buoyancy lag time is determined to assess the time taken by the dosage form to float on the top of the dissolution medium after it is placed in the medium. These parameters can be measured as a part of the dissolution test.

\section{Floating time}

The time for which the dosage form continuously floats on the dissolution media is termed as floating time. It is usually performed in Simulated Gastric Fluid maintained at $37^{\circ} \mathrm{C}$ [31].

\section{Swelling systems}

Swelling Index: After enthrallment of dosage form into Simulated Gastric Fluid at $37{ }^{\circ} \mathrm{C}$, the dosage form is removed out at regular interval and dimensional changes are measured in terms of merger in tablet thickness/diameter with time.

\section{In vivo evaluation: radiology}

Barium Sulphate is widely used as Radio Opaque Marker. X-ray is used for examination of internal body systems. So, $\mathrm{BaSO}_{4}$ is fused inside dosage form and X-ray images are taken at various intervals to view gastric retention.

\section{Gastroscopy}

Gastroscopy is used to inspect visually the effect of prolongation in the stomach. Scintigraphy: Like X-ray, emitting materials are incorporated into dosage form and then images are taken by scintigraphy. Widely used emitting material is 99 Tc.

\section{Ultrasonography}

It is not used generally because it is not traceable at intestine. 


\section{Magnetic marker monitoring}

This technique is radiationless and so not hazardous. In this technique, dosage form is magnetically marked by incorporating iron powder inside, and images can be taken by very sensitive bio-magnetic measurement equipment. In vivo evaluation of gastric retention Analysis of the position of the dosage form in the GIT involves an imaging technique such as $\gamma$-scintigraphy and X-ray [32]. In $\gamma$-scenographer, a small amount of stable isotope is compounded in the dosage forms during its preparation. The admittance of a $\gamma$-emitting radio-nuclide in a formulation allows indirect external observation using a $\gamma$-camera or scinti scanner. For $\mathrm{x}$-ray, barium sulfate is used as a contrast medium. It helps to uncover dosage form in the GIT by which one can predict and correlate the gastric emptying time and the passage of dosage form. In gastro-scope and ultrasonography studies can be included in the in vivo evaluation of GRDDS. Gastro-scope comprises of peroral endoscopy, used with a fiber optic and video systems. Ultra-sonography is not routinely used in the evaluation of GRDDS. In vivo plasma profile can also be obtained by operating the study in suitable animal model. Water uptake study: when the dosage form in simulated gastric fluid at $37^{\circ} \mathrm{C}$ and determining the dimensional changes, such as diameter and thickness, at regular interval of time. After some time, the swollen tablets are weighed and water uptake is measured in terms of percentage weight gain, as given:

\section{Entrapment efficiency}

The drug is extracted by suitable method and analyzed to find out the amount of drug present [34,35].

\section{CONCLUSION}

Gastroretentive drug delivery system has emerged as an efficient means of prolonged retaining ability in the stomach and thereby increase gastric residence time of drugs and improves bioavailability of drugs. Despite the number of difficulties to be worked out to achieve prolonged gastric retention, many companies are focusing towards commercializing this technique. Number of commercial products and patents issued in this field are evident of it.

\section{ACKNOWLEDGEMENT}

Famotidine was obtained as a gift sample from Srinivasan Pharmaceuticals Pvt. Ltd, Hyderabad, for providing the gift sample of Famotidine

\section{FUNDING}

Nil

\section{AUTHORS CONTRIBUTIONS}

All the authors have contributed equally.

\section{CONFLICT OF INTERESTS}

The authors declare no conflict of interest.

\section{REFERENCES}

1. Sharma AR, Khan A. Gastro-retentive drug delivery system: an approach to enhance gastric retention for prolonged drug release. Indian J Pharm Sci 2014;5:1095.

2. Gupta P, Kesh M. Formulation, and evaluation of gastroretentive floating tablets. Int J Pharm 2018;6:1.

3. Pandey A, Kumar G, Kothiya P, Brasilia Y. A review of current approaches in the gastro retentive drug delivery system. Asian J Pharm Health Sci 2012;2:139-46.

4. Sandhya P, Farhat A, Benaziya F, Durani HZ. A review of the gastro retentive drug delivery system of helicobacter pylori. Int J Pharma Res Rev 2013;1:403.

5. Rathod HJ, Mehta DP, Yadav JS. A review on gastroretentive drug delivery systems. Pharma Tutor 2016;4:29-40.

6. Badoni A, Ojha A, Gnanarajan G, Kothiyal P. Review on gastro retentive drug delivery system. Pharma Innov 2012;18, Part A):32.

7. Makwana A, Sameja K, Parekh H, Pandya Y. Advancements in controlled release gastroretentive drug delivery system: a review. Pharma Innov 2012;2:12-3.
8. Nayak KP, Upadhyay P, Valera AR, Chauhan NP. Gastroretentive drug delivery systems and recent approaches: a. J Pharm Res Opinion 2012;2:1-8.

9. Kagan L, Hoffman A. Selection of drug candidates for gastroretentive dosage forms: pharmacokinetics following continuous intragastric mode of administration in a rat model. Eur J Pharm Biopharm 2008;69:238-46.

10. Reddy LH, Murthy RS. Floating dosage systems in drug delivery. Crit Rev Ther Drug Carrier Syst 2002;19:553-85.

11. Hoffman A. Pharmacodynamic aspects of sustained-release preparations. Adv Drug Delivery Rev 1998;33:185-99.

12. Huang Y, Leobandung W, Foss A, Peppas NA. Molecular aspects of muco-and bioadhesion: tethered structures and site-specific surfaces. J Controlled Release 2000;65:63-71.

13. Hooda A, Nanda A, Jain M Kumar, Rathee P. Optimization and evaluation of gastroretentive ranitidine $\mathrm{HCl}$ microspheres by using design expert software. Int J Biol Macromol 2012;51:691-700.

14. Pawar HA, Dhavale R. Development, and evaluation of gastroretentive floating tablets of an antidepressant drug by thermoplastic granulation technique. Beni-Suef University J Basic Appl Sci 2014;3:122-32.

15. Vyas SP, Khar RK. Gastro retentive system in controlled drug delivery: concepts and advances. Nayal AS, Pandey S, Gnanarajan G. Review: an overview on gastro retentive floating tablet. Int J Pharm Chem Sci 2013;2:1357-65.

16. Sravya K, Kavitha K, Rupeshr M, Jagdeesh Singh. Gastroretentive drug delivery systems: a review. Res J Pharm Biol Chem Sci 2012;3:966-80.

17. Machete H, Patel MR, Patel KR, Patel MS. An overview of floating drug delivery system. Pharma Tutor 2014;2:61-71.

18. Jamil F, Kumar S, Sharma S, Vishwakarma PS, Singh L. Review on stomach specific drug delivery systems: development and evaluation. Int J Res Pharm Biomed Sci 2011;2:142-71433.

19. Patel N, Nagesh C, Chandrashekhar S, Jinal P, Devdatt J. Floating drug delivery system: an acceptable innovative approach in gastroretentive drug delivery. Res J Pharm Dosage Forms Technol 2012;4:93-103.

20. Makwana A, Sameja K, Parekh H, Pandya Y. Advancements in controlled release gastroretentive drug delivery system: a review. J Drug Delivery Ther 2012;2:44-5.

21. Pilgaonkar PS, Rustomjee MT, Gandhi AS. Inventors; Rubicon Res Pvt Ltd, assignee. Gastroretentive dosage forms of GABA analogs. U. S patent application, US 13/700,794; 2013.

22. Chawla G, Bansal A. A means to address regional variability in intestinal drug absorption. Pharm Tech 2003;27:50-68.

23. Sheth PR, Tossounian J. The hydrodynamically balanced system $\left(\right.$ HBS $^{\mathrm{TM}}$ ): a novel drug delivery system for oral use. Drug Dev Ind Pharm 1984;10:313.

24. Arora S, Ali J, Ahuja A, Khar RK, Baboota S. Floating drug delivery systems: a review. AAPS PharmSciTech 2005;6:372-90.

25. Prakash B, Neelima K, Snehith S, Ramesh C. Development of gastro retentive drug delivery system. ARS Pharmaceutica 2009;5:8-24.

26. Watts J, Smith A, Bond J, Lafferty W. Floating drug delivery composition. WO/2001/058424; 2001.

27. Vyas SP, Khar RK. Gastroretentive systems. In: Controlled drug Delivery. Vallabh Prakashan. Delhi, India; 2006. p. 197-217.

28. Clarke GM, Newton JM, Short MD. Gastrointestinal transit of pellets of differing size and density. Int J Pharm 1993;100:81-92.

29. Moes AJ. Gastric retention systems for oral drug delivery. Business Briefing: Pharmatech; 2003. p. 157-9.

30. Vedha Hari BN. The recent developments on gastric floating drug delivery systems: an overview. Int J Pharmtech Res 2010;2:524-34.

31. Rednick AB, Tucker SJ. Sustained-release bolus for animal husbandry, US patent US; 1970;3:507-952.

32. Geetha A, Kumar J Rajendra, Mohan CH Krishna. Review on: floating drug delivery systems. Int J Pharm Res Biomed Anal 2012;1:1-13.

33. Choi BY, Park HJ, Hwang SJ, Park JB. Preparation of alginate beads for floating drug delivery system effects of $\mathrm{CO}_{2}$ gasforming agents. Int J Pharm 2002;239:81-91.

34. Ichikawam, Watenables, Miyake Y. A multiple-unit oral floating dosage systems preparation and in vivo evaluation of floating and sustained-release characteristics. J Pharm Sci 1991;80:1062-6. 